\title{
Surgical treatment of aneurysmal changes in the ascending aorta
}

\author{
VIK ING OLOV B JÖRK A N D LAR S B J ÖR K \\ From the Departments of Thoracic Surgery and Diagnostic Radiology, University Hospital. \\ Uppsala. Sweden
}

The pioneer work on the surgical treatment of aneurysm of the ascending aorta was performed by Bahnson and Nelson (1956). They emphasized that by aneurysmorrhaphy and narrowing the base of the aorta the aortic insufficiency was relieved at the same time. With an experience of four cases, we consider concomitant aortic insufficiency the most important part of this disease.

\section{CASE REPORTS}

CASE 1 A 51-year-old man experienced sudden pain in the chest and neck. The pain radiated along the vertebral column down to the right leg. which was paralysed. The patient lost consciousness. The symptoms disappeared slowly and he tried to return to work. He then developed cardiac decompensation. fluid retention. and oedema. One year after the first onset of the acute attack the patient developed progressive symptoms of precordial pain and dyspnoea. Physical examination revealed both systolic and diastolic murmurs over the base of the heart and several extrasystoles. Radiological investigation showed an enlarged heart of a total of $1.770 \mathrm{ml}$., corresponding to $840 \mathrm{ml} . / \mathrm{sq}$. m. body surface area. Aortography showed a grade IV aortic insufficiency (Fig. 1), an aneurysm of the ascending aorta. and a dissecting aortic aneurysm. The patient was accepted for surgery on 27 April 1961.

Median sternotomy was performed and the heart was connected to a heart-lung bypass with a total perfusion of 143 minutes and aortic occlusion for 130 minutes. During this time there was a left coronary artery perfusion of 112 minutes and a right coronary artery perfusion of 47 minutes. Both the left and right coronary cusps were found to be prolapsing. but the non-coronary cusp was considered to be intact. An internal bicuspidalization was performed with approximation of the left and noncoronary cusps. The ascending aneurysm was excised and from the dissection the inner tube was approximated to the outer tube distally, as well as centrally. just above the coronary arteries. A dacron graft was then placed first postero-centrally, using one row of interrupted mattress sutures and one row of continuous 3-0 silk. Then the distal part of the prosthesis was connected to the aorta, first posteriorly and then $\vec{\omega}$ anteriorly. Lastly the central anterior part was con- $\tilde{N}$ nected to the base of the aorta. which permitted $\overrightarrow{0}$ coronary perfusion to be continued while the last 음 few mattress stitches were inserted. Pressure necrosis from the aneurysm on the wall of the pulmonary artery had caused a weak area that ruptured and had to be repaired. The heart took over the circulation well, and the patient recovered from the operation without complications.

The post-operative course was complicated by fever and a blood culture positive for Staphylococcus aureus. A month after the operation the diastolic aortic murmur returned and the patient began to go

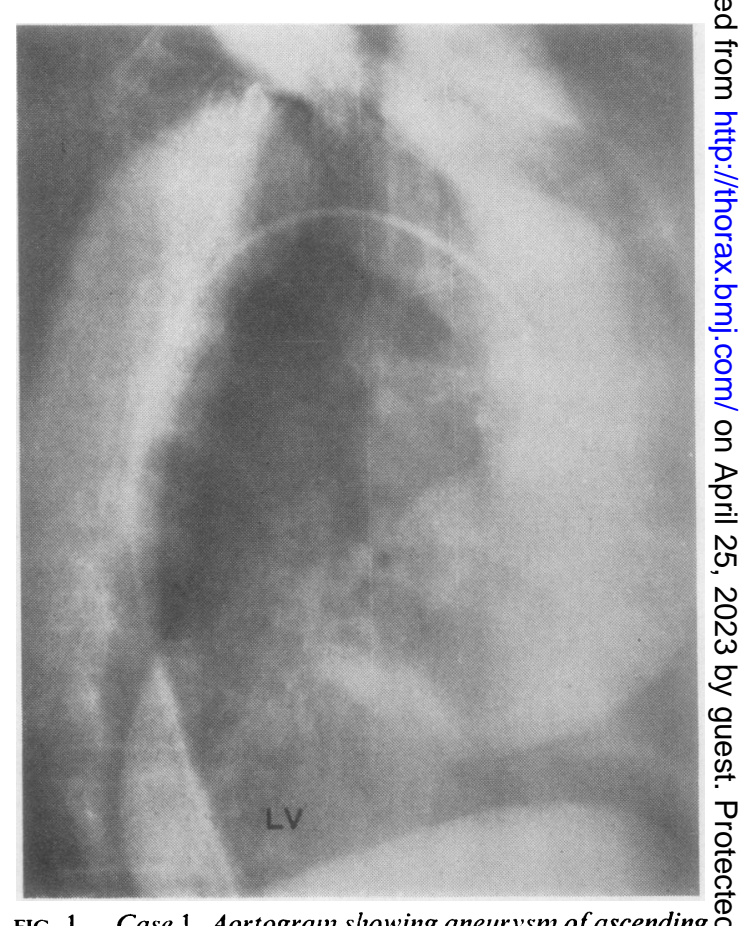

FIG. 1. Case 1. Aortogram showing aneurvsm of ascending $\stackrel{\Phi}{\varrho}$ aorta and aortic insufficiency with filling of the left ventricle (LV). 
downhill with heart failure and multiple emboli. He died on 17 June 1961 with sepsis, recurrent aortic insufficiency, and cardiac infarction. Necropsy revealed that one of the sutures anteriorly had constricted the orifice of the right coronary artery, thereby causing an occlusion and a large infarct. The sutures in the aortic cusps for internal bicuspidalization had cut through with recurrent aortic insufficiency. The aortic graft was intact. Examination of the aneurysmal wall showed severe degeneration with necrosis, i.e., idiopathic medial necrosis.

CASE 2 A 46-year-old woman had a two years' history of aortic insufficiency; her heart had increased in size from $550 \mathrm{ml}$. to $690 \mathrm{ml}$./sq. m. body surface area during this time. Aortography showed a huge dilatation of the ascending aorta and aortic insufficiency of grade III. The whole of the left ventricle was filled with contrast injected into the aorta, but the density of contrast in the left ventricle was never the same as that in the aorta (Fig. 2a). A left ventricular angiogram showed good left ventricular contractions. At operation through a median sternotomy the total perfusion time was 72 minutes and aortic occlusion lasted for 67 minutes. The left coronary artery was perfused

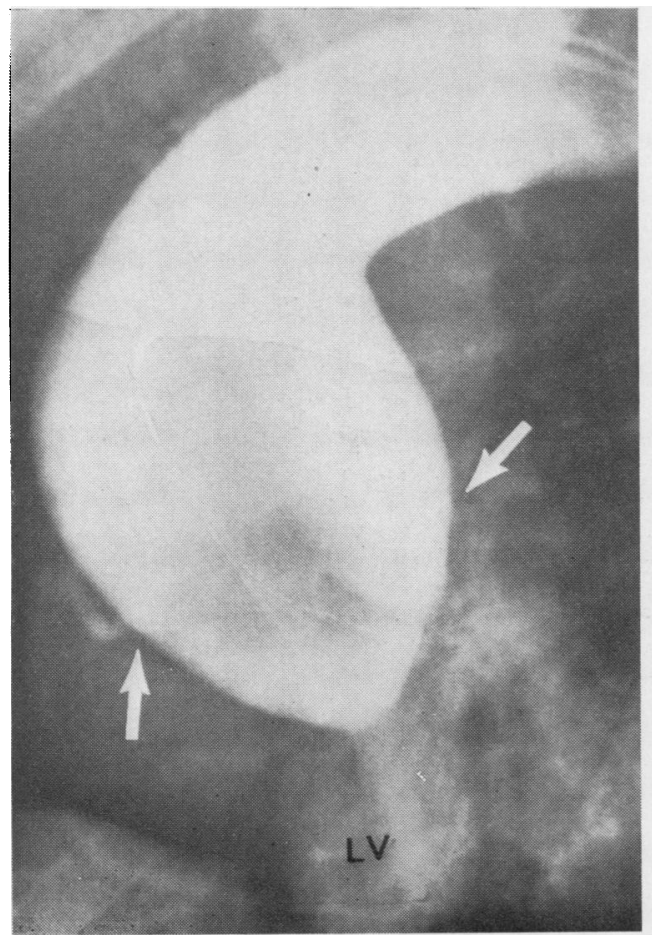

FIG. 2a for 58 minutes and the right coronary artery for 60 minutes. All three cusps were prolapsing and were excised, after which a no. 10 ball valve prosthesis was introduced with 15 mattress sutures, all sutures being buffered by a teflon pearl on the ventricular side and all sutures being tied on the inside of the aorta. When the ball valve prosthesis had been sutured in position the thin areas of the aneurysm were excised and an aneurysmorrhaphy was performed. The patient made an uneventful recovery. One month later the heart had diminished in size to $590 \mathrm{ml} . / \mathrm{sq}$. m. body surface area. Pathological examination of the specimen showed idiopathic cystic medial necrosis.

At follow-up 12 months after operation the patient's general condition was good, the heart had diminished in size to $580 \mathrm{ml} . / \mathrm{sq}$. m. body surface area, and there was no diastolic murmur. The patient could perform an exercise tolerance test of 500 kilopondmeters/ minute. Aortography (Fig. 2b) showed approximately the same size of aortic aneurysm as before aneurysmorrhaphy but a competent aortic prosthesis.

CASE 3 A 34-year-old man known to have had heart failure since birth complained of pain in the heart

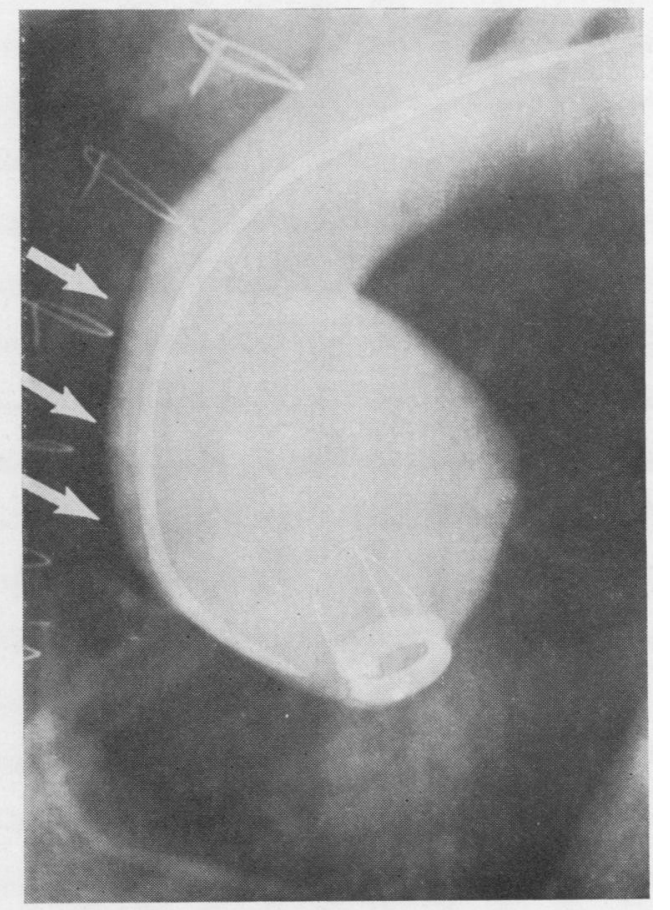

FIG. $2 b$

FIG. 2. Case 2 (a) Pre-operative aortogram showing an aneurysm of the ascending aorta, prolapsing cusps, and aortic insufficiency with filling of the left ventricle $(\mathrm{LV})$. The coronary artery ostia are displaced upwards (arrows). (b) Aortogram nine months post-operatively showing a competent ball valve prosthesis and some flattening of the anterior contour of the aorta at the site of aneurysmorrhaphy (arrows). Width of aorta otherwise unchanged. 

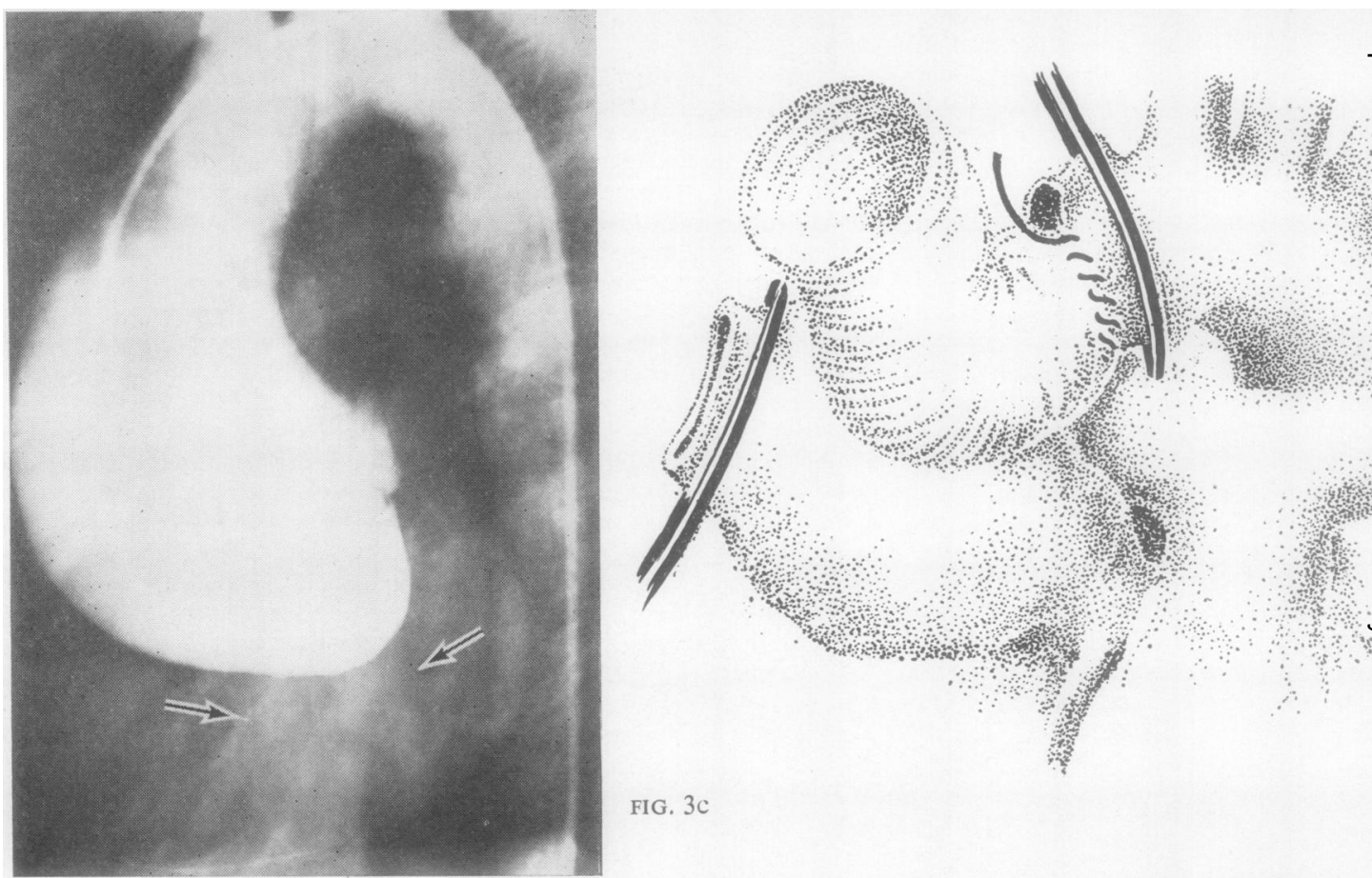

FIG. 3c

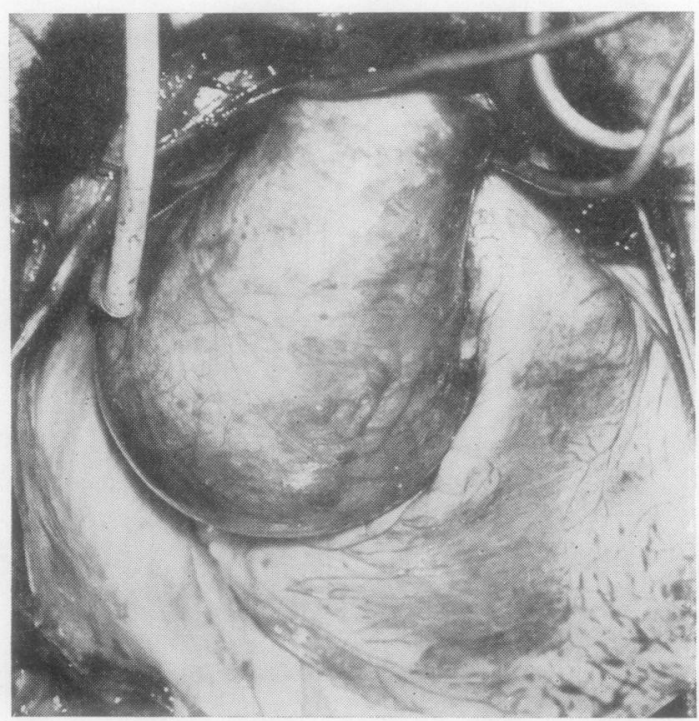

FIG. $3 b$ 


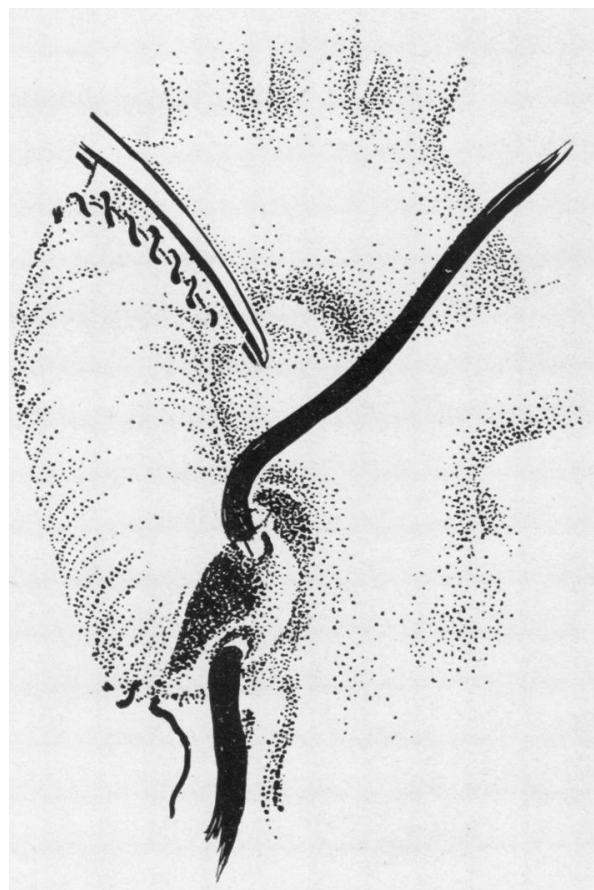

FIG. 3e

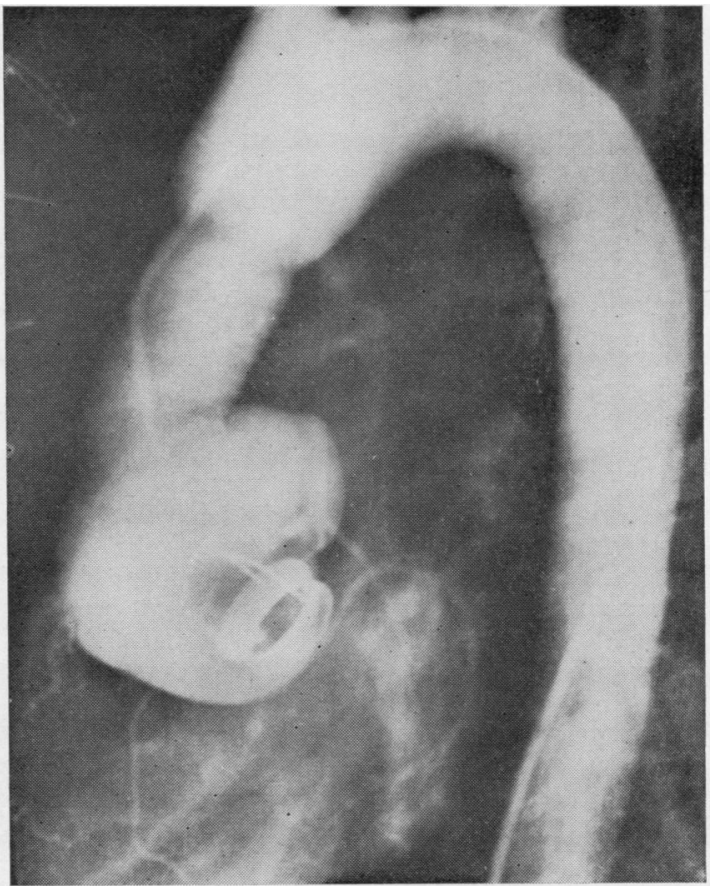

FIG. $3 \mathrm{~g}$

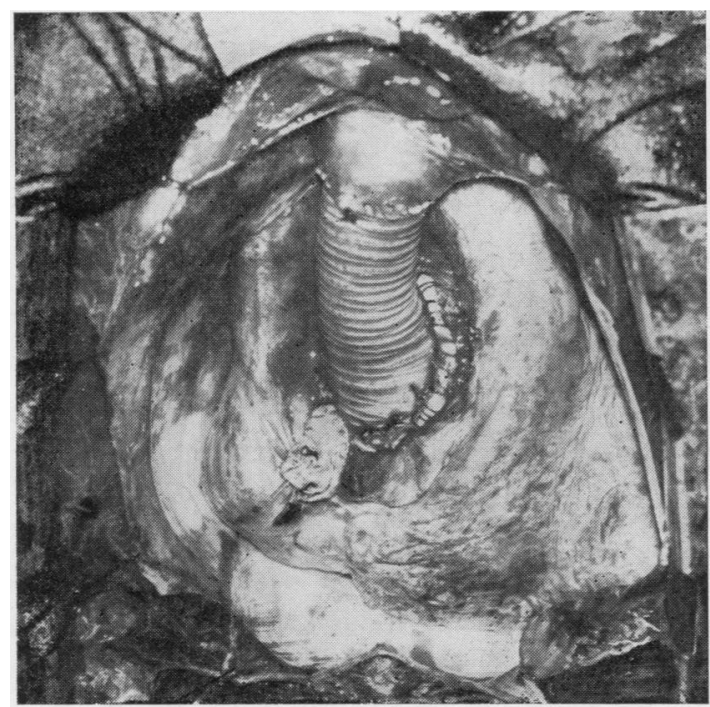

FIG. 3. Case 3 (a) Aortogram showing an aneurysm of the ascending aorta, prolapsing cusps, and aortic insufficiency (arrows). (b) Photograph taken at operation shows a huge aneurysm of the ascending aorta. (c) The first step with division of the ascending aorta distal to the aneurysm. The distal suture line of the dacron graft to the aorta is completed while coronary perfusion is provided by the beating left ventricle. (d) The central posterior suture line. Both right and left coronary artery perfusion is carried out during fixation of the ball valve prosthesis. All fixation sutures are placed within the aorta. (e) The completion of the central anterior suture line between the graft and the aorta. Coronary perfusion is continued until the anterior central suture is completed with interrupted mattress sutures. (f) The dacron graft in the ascending aorta. (g) Aortogram eight months post-operatively shows ball valve prosthesis and dacron prosthesis in ascending aorta. Width of aortic root and aorta above dacron prosthesis is unchanged. 
and palpitation on exertion. Physical examination revealed a systolic murmur over the base of the heart continuing up over the carotid arteries and a diastolic murmur to the left of the sternal border. Radiographs showed a normal cardiac contour with a total volume of $760 \mathrm{ml}$. corresponding to a relative volume of $480 \mathrm{ml}$./sq. m. body surface area. Thoracic aortography showed dilatation of the ascending aorta with a diameter of more than $10 \mathrm{~cm}$. Significant aortic insufficiency was also demonstrated (Fig. 3a).

The patient was operated on through a median sternotomy (Fig. 3b). He was connected to the heartlung machine for 116 minutes. The aorta was occluded for 108 minutes: it was first divided between clamps just central to the innominate artery. The left ventricle was drained and only the tape around the inferior vena cava was occluded around the drainage tube. Thus there was a return of blood through the superior vena cava, and then the left ventricle was drained only so much that an adequate coronary perfusion through both coronary arteries was provided through the aorta for the first 23 minutes (Fig. 3c). Then the aneurysm was excised : the left coronary artery was perfused for 83 minutes and the right coronary artery for 65 minutes. The commissure between the non-coronary and the right coronary cusp was fused and calcified. The commissurotomy between the left and right coronary cusps was also fused. A commissurotomy would only have increased the already significant aortic insufficiency. The aortic cusps were therefore excised and a no. 11 StarrEdwards ball valve prosthesis was sutured in position with 15 interrupted mattress sutures buffered with teflon pearls. During the first part of the procedure. when the coronary arteries were perfused directly through the aorta, the distal suture between the prosthesis and the aorta was performed using a continuous 3-0 suture to a dacron prosthesis of $2 \cdot 5 \mathrm{~cm}$. diameter. After the ball valve prosthesis had been sutured in position the central part of the anastomosis was performed, first posteriorly and then anteriorly (Fig. 3d, e, and f). The heart took over the circulation well. A $10 \mathrm{~mm}$. $\mathrm{Hg}$ gradient was measured over the aortic prosthesis. The patient made an uneventful recovery. Pathological investigation demonstrated an idiopathic cystic medial necrosis and calcified aortic cusps. The patient has been followed for 10 months.

At follow-up the patient had experienced several periods of arrhythmia and had not returned to work. There was no diastolic murmur and the patient could perform an exercise tolerance test of 400 kilopondmeters/minute. At aortography (Fig. 3g) a competent aortic valve prosthesis was found and a normally functioning ascending aortic graft. The heart had diminished in size to a total of $550 \mathrm{ml}$., corresponding to $350 \mathrm{ml}$./ sq. m. body surface area.

CASE 4. A 48-year-old man had a syphilitic aneurysm of the ascending aorta as well as a large aneurysm of the arch of the aorta with tracheal compression. The patient underwent tracheostomy (a long plastic

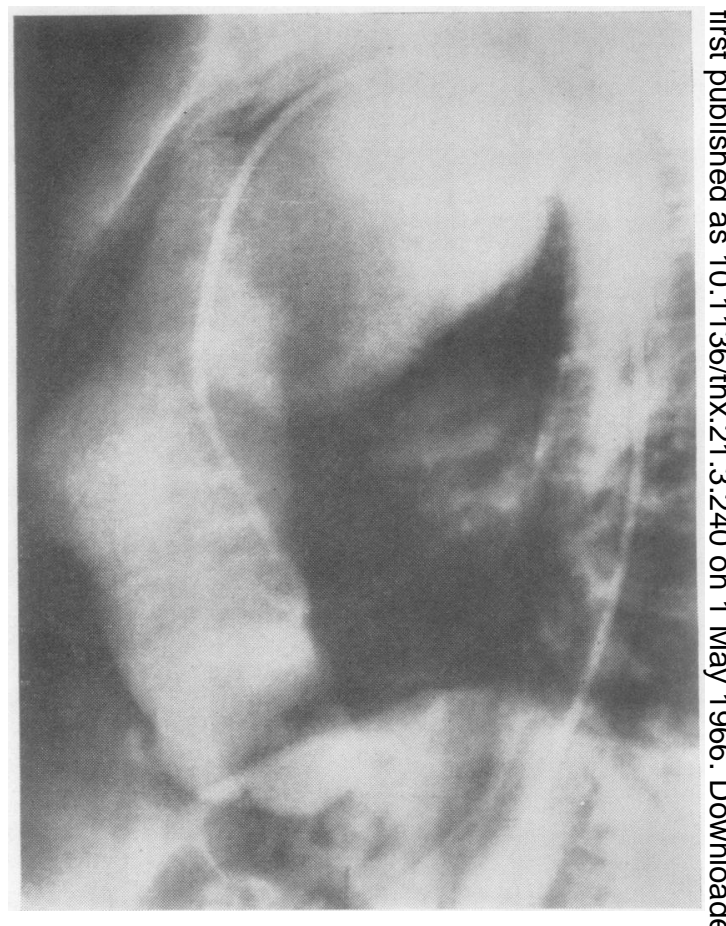

FIG. 4. Case 4. Aortogram showing aneurysm of the ascending aorta and arch. No aortic insufficiency.

intratracheal tube was used) as well as an exploratory thoracotomy six months before surgery. Aortography우 showed competent aortic cusps (Fig. 4). On 11 September 1962 the ascending aorta and the whole aortic음 arch were excised and replaced by a graft with side branches to the innominate artery as well as to the left carotid. The left subclavian artery was $\underline{3}$. anastomosed to the graft (Fig. 5). The case has been reported in the Journal of Thoracic and Cardio-윽 vascular Surgery (1963). 45. 817. The patient recovered and has now been followed for two and a half years. He continues to be in good general health. $\frac{D}{0}$

\section{DISCUSSION}

In three of our four patients the ascending aortic $\mathcal{O}^{\circ}$ aneurysm was caused by idiopathic cystic medial necrosis, which is a non-inflammatory degenera-O tive process. This condition is commonest in thee ascending aorta. We have encountered one case of aneurysm of the descending aorta as well as? one of aneurysm of the subclavian artery due to 0 idiopathic cystic medial necrosis. In one of our $\frac{\vec{\Phi}}{\mathbb{D}}$ patients the lesion had caused a dissecting aneurysm. The other two were fusiform $\mathbb{\complement}$ aneurysms of the ascending aorta. As a consequence of focal degeneration of the elastic 


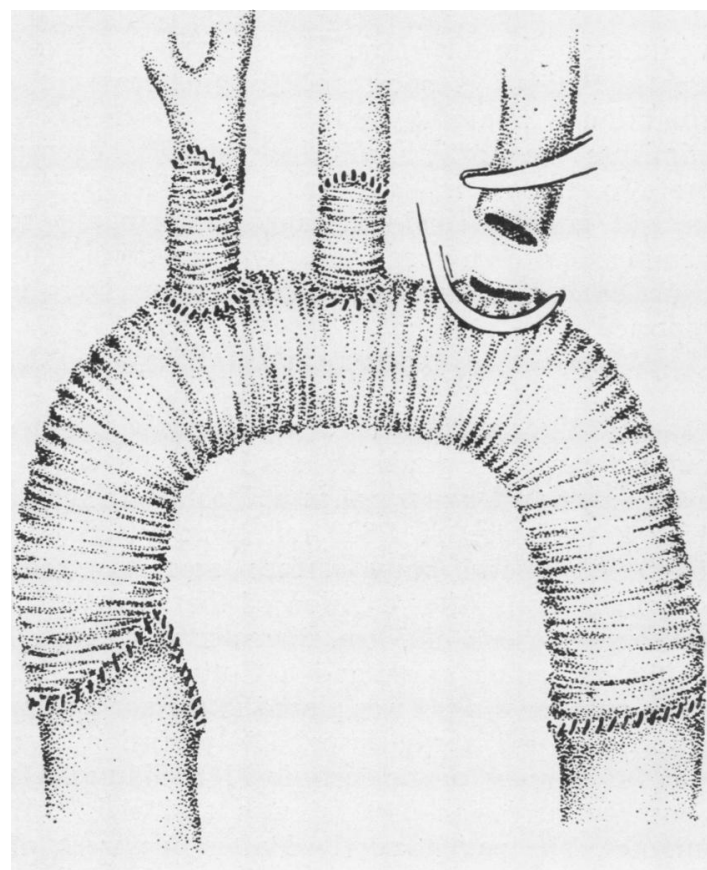

FIG. $5 \mathrm{a}$

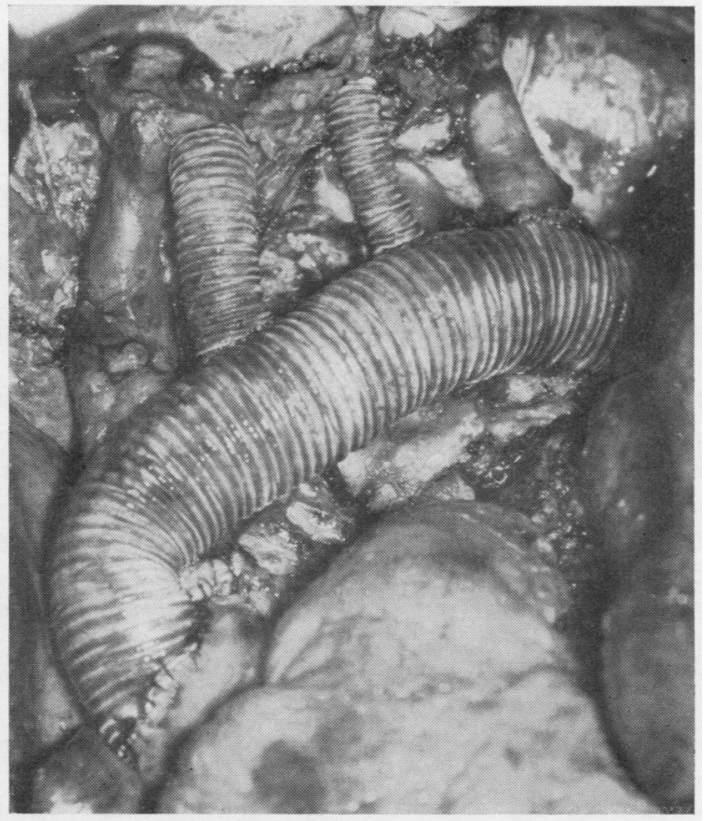

FIG. 5 b

FIG. 5. Case 4 (a) Diagram of the operation with a total aortic arch replacement. (b) Shows a total aortic arch replacement with a dacron graft.

tissue of the media a dilatation of the aorta can develop. The intima is not involved in the disease and may not dilate with the media, so that a rent occurs in the intima causing a dissecting aneurysm, with a channel between the outer and middle thirds of the media. In cases of fusiform aneurysm the intima has remained adherent to the underlying media; as the aneurysm has enlarged the lumen has become lined with large patches of bare media. The process extends right back to the attachment of the aortic valve leaflets, and in all our three patients there was aortic insufficiency and in one aortic stenosis. The stretching of the aortic wall central to the coronary ostia may displace them as much as 3 or $4 \mathrm{~cm}$. distally to the aortic valve ring. Pathological investigation demonstrated idiopathic cystic medial necrosis in all three patients. Bahnson suggested the possibility of correcting the aortic insufficiency by decreasing the size of the aortic ring when excising the ascending aortic aneurysm. From case 3 (Fig. $3 \mathrm{~g}$ ) it is obvious that a narrowing of the ascending aorta above the coronary arteries cannot significantly influence the sinus of Valsalva and the aortic cusps, and therefore an aortic ball valve prosthesis must be used to correct the aortic insufficiency. It is our experience as well as that of others that a concomitant aortic insufficiency is the most serious complication needing treatment. In our case 1 an internal bicuspidalization was performed with a good immediate result. Due to bloodstream infection the aortic insufficiency recurred and the patient died. In cases 3 and 4 we chose to introduce a ball valve prosthesis. In one an aneurysmorrhaphy was used, in the other a dacron graft. The question arises whether this disease is progressive and whether a progressive dilatation of the ascending aorta will occur. If it does all aneurysms should be excised and replaced by a dacron graft. If, however, the aneurysmorrhaphy is found to give as good a late result as a dacron graft in the ascending aorta in cases with idiopathic cystic medial necrosis, this method should be used on suitable occasions, because it is quicker and better tolerated. The seriousness of this disease is obvious from one report of six deaths in 14 patients treated surgically.

Regarding the surgical technique the steps taken in case 3 seem to be of advantage, i.e., first the ascending aorta was divided proximal to the innominate artery. As the patient was on bypass without occlusion of the tapes around the caval catheters enough blood passed through the lungs 
into the left ventricle to give good coronary perfusion during this first step. A drainage catheter in the left ventricle regulated the pressure in the ascending aortic aneurysm, thus securing coronary artery perfusion. During this time the distal end of the graft was anastomosed to the aorta with continuous 3-0 sutures. As a second step the aneurysm was removed, the coronary arteries were cannulated and perfused while a ball valve prosthesis was sutured in place. We always prefer to place the fixation sutures within the aorta but have the 0 silk sutures buffered by teflon pearls to prevent them cutting through. If it is difficult to decide whether an aneurysmorrhaphy should be performed or a graft introduced, the aneurysm must be opened first and the coronary arteries cannulated at once. Resection of an ascending aortic aneurysm must be done with care. In one patient we encountered a pressure necrosis of the pulmonary artery, and in retrospect it would have been wiser to leave the part of the aneurysm against the pulmonary artery in situ. In judging which operation should be performed, aneurysmorrhaphy or a dacron graft, it may be recalled that all the diseased aortic wall can never be removed, as it would be too time-consuming and dangerous to remove the subcoronary part of the aorta, i.e., the part between the aortic leaflets and the coronary ostia. Furthermore, it is possible that by introducing a ball valve prosthesis and eliminating the aortic insufficiency there will be no progression of the aortic dilatation. We at present prefer a dacron graft together with a ball valve prosthesis in those patients whom we judge able to survive that procedure, and we reserve aneurysmorrhaphy for older patients whose general condition is not good. Whether the degenerative process progresses or becomes arrested after surgery remains to be seen.

\section{SUMMARY}

Ascending aortic aneurysm is usually due to idiopathic cystic medial necrosis, and it is combined with aortic insufficiency. The prognosis of un $-\frac{\vec{F}}{\vec{s}}$ treated patients is poor, and for this reason the condition is treated by a ball valve prosthesis, to등 eliminate the aortic insufficiency, and a dacron $\bar{c}$. graft for the ascending aorta. In an older patient $\widehat{\nabla}$ in bad general condition, aneurysmorrhaphy is combined with an aortic ball valve prosthesis. The experience of four cases with three survivors is $\overrightarrow{0}$ reported.

\section{ADDENDUM}

CASE 5 Since submission of this paper a 59 -year- $-x$ old woman with grade IV aortic insufficiency $+\stackrel{\sim}{-}$ ascending aortic aneurysm as well as a giant heart $\omega$ with a total size of $1,800 \mathrm{ml}$. corresponding to $1,180 \mathrm{ml} . / \mathrm{sq}$. $\mathrm{m}$. body surface area has been operated upon. After excision of the aneurysm a? dacron prosthesis of $3 \mathrm{~cm}$. diameter was inserted $\vec{Z}$ in the ascending aorta and a Cutter aortic ball valve prosthesis no. 5 was placed in the aorta after excision of the aortic cusps. The perfusion time was 143 minutes, aortic occlusion 116 minutes, left. coronary perfusion 110 minutes and right $107 \mathrm{O}$ minutes. The patient survived after one week of treatment in a respirator.

\section{BIBLIOGRAPHY}

Bahnson, H. T., and Nelson, A. R. (1956). Cystic medial necrosis as a⿳亠二口 cause of localized aortic aneurysms amenable to surgical treat-3 ment. Ann. Surg., 144, 519.

and Spencer, F. C. (1960). Excision of aneurysm of the ascending aorta with prosthetic replacement during cardiopulmonary by= pass. Ibid., 151, 879 .

Björk, V. O. (1963). Successful replacement of the total aortic arch for aneurysm. J. thorac. cardiovasc. Surg., 45, 817.

Bosher, L. H., Brooks, J. W., and Pois, A. J. (1964). The surgica treatment of dissecting aneurysm of the ascending aorta: with ax report of four cases in the chronic stage. Ann. Surg., 159, 829. व

Cooley, D. A., and DeBakey, M. E. (1956). Resection of entire ascending aorta in fusiform aneurysm using cardiac bypass. $J$ Amer. med. Ass., 162, 1158.

De Bakey, M. E., Cooley, D. A., and Creech, O., Jr. (1955). Surgica? considerations of dissecting aneurysm of the aorta. Ann. Surg., 142, 586 .

Groves, L. K., Effler, D. B., Hawk, W. A., and Gulati, K. (1964), 극 Aortic insufficiency secondary to aneurysmal changes in the ascending aorta: Surgical management. $J$. thorac. cardiovas $(20$ Surg., 48, 362.

Spiekerman, R. E. and McGoon, D. C. (1960). Aneurysm of the ascending aorta with obstruction of the superior vena cava: ascending aorta with obstruction of the superior vena cava: Dis. Chest, 37, 675. 